\title{
Association between healthy lifestyle score and breast cancer
}

\author{
Batoul Ghosn?', Sanaz Benisi-Kohansal', Soraiya Ebrahimpour-Koujan', Leila Azadbakht ${ }^{1}$ and \\ Ahmad Esmaillzadeh $2,1,3^{*}$
}

\begin{abstract}
Background: Majority of earlier studies have assessed the association between individual lifestyle factors and the risk of breast cancer (BC); however, limited information is available linking the whole lifestyle factors to $\mathrm{BC}$. We aimed to examine the association between combined lifestyle score (diet, physical activity (PA) and smoking) and risk of BC in Iranian population.

Methods: This population-based case-control study included 350 newly diagnosed cases of BC and 700 healthy controls randomly selected from adult women. Dietary intakes, PA and smoking status of study participants were examined using validated questionnaires. The lifestyle risk factors examined in this study included cigarette smoking, physical inactivity, and Healthy Eating Index-2010 (HEl-2010). The lifestyle score ranged from zero (nonhealthy) to 3 (most healthy) points. Logistic regression models were fitted to investigate the association between combined lifestyle scores and odds of BC.
\end{abstract}

Results: Mean age and body mass index (BMI) of study participants were 62.4 years and $24.3 \mathrm{~kg} / \mathrm{m}^{2}$, respectively. In the whole study population, individuals with the highest healthy lifestyle score (HLS) were 0.38 times less likely to have $\mathrm{BC}$ than those with the lowest score (OR: 0.62; $\left.95 \% \mathrm{Cl}: 0.40,0.93, P_{\text {trend }}=0.01\right)$. The analysis by menopausal status showed that postmenopausal women with the highest HLS had $44 \%$ lower odds of BC compared with those with the lowest score (OR: 0.56; $\left.95 \% \mathrm{Cl}: 0.36,0.88, P_{\text {trend }}=0.004\right)$. Such association was not seen in premenopausal women. After analyzing each component of HLS, we found that individuals with the highest HEI score were $46 \%$ less likely to have BC than those with the lowest score (OR: 0.54; 95\% Cl: 0.35, 0.82, $\left.P_{\text {trend }}<0.001\right)$. No other significant associations were found between PA and smoking and risk of BC.

Conclusions: Significant inverse associations were found between HLS and HEl with BC especially among postmenopausal women. Prospective studies are required to confirm these findings.

Keywords: Diet, Physical activity, Smoking, Lifestyle, Breast cancer

\section{Introduction}

Breast cancer $(\mathrm{BC})$ is highly prevalent in the world, particularly in developing countries. Incidence rates differ widely throughout the world, from 27 per 100,000 in Middle Africa and Eastern Asia to 92 per 100,000 in Northern America [1]. In Iran, BC is the fifth most

\footnotetext{
* Correspondence: a-esmaillzadeh@tums.ac.ir

${ }^{2}$ Obesity and Eating Habits Research Center, Endocrinology and Metabolism Molecular- Cellular Sciences Institute, Tehran University of Medical Sciences, Tehran, Iran

${ }^{1}$ Department of Community Nutrition, School of Nutritional Sciences and Dietetics, Tehran University of Medical Sciences, P.O. Box 14155-6117, Tehran, Iran

Full list of author information is available at the end of the article
}

common causes of death related to cancer comprising $24.4 \%$ of all cancers with age standardized rate (ASR) of 23.1 per 100,000 [2]. The national database revealed a progressive increasing trend in the cumulative probability of $\mathrm{BC}$ incidence for individuals aged $15-79$ years in Iran in the last 30 years [3].

Several modifiable risk factors including overweight or obesity [4], alcohol use [5], physical inactivity [6] and prolonged steroid hormones exposure [7] have been linked to BC. Non-modifiable risk factors include increasing age [8], positive family history of $\mathrm{BC}$ [9] and reproductive factors [8]. There is currently limited evidence that diet is related to $\mathrm{BC}$. In a review on

(c) The Author(s). 2020 Open Access This article is distributed under the terms of the Creative Commons Attribution 4.0 International License (http://creativecommons.org/licenses/by/4.0/), which permits unrestricted use, distribution, and 
prospective epidemiologic studies, Michels et al. failed to find any significant association between diet and $\mathrm{BC}$ [10]. However, the American Cancer Society recommendations regarding healthy lifestyle include eating a healthy diet, getting at least 150 min of moderate intensity exercise each week, avoiding smoking and keeping low stress levels [11]. Thus, to reduce the risk, it is important to determine the degree to which women are adhering to these recommendations.

There are few studies published on the link between healthy lifestyle index and BC. Arthur et al. found a 30\% higher risk of $\mathrm{BC}$ among those in the lowest category (0-10 points) of the healthy lifestyle score (HLS) compared to those in the highest category ( $\geq 15$ points) [12]. Similarly, Sanchez-Zamorano et al. concluded that healthy lifestyle was associated with a decreased risk of having $\mathrm{BC}$ [13]. However, some other studies failed to find any association [14]. It must be kept in mind that these studies were conducted in western countries and to our knowledge, no study was done in eastern countries, specifically Iran, investigating the healthy lifestyle factors in relation to risk of BC. Given the limitations in the construction of HLS, inconsistent findings and small number of cases in previous studies, generalization of their findings might be limited.

We examined the contribution of whole lifestyle to the risk of $\mathrm{BC}$ because of these important factors: (1) the different pattern of diet and physical activity (PA) in developing countries compared with those in western nations, (2) BC being the most prevalent cancer among Iranian women. Therefore, this study was done to assess the association between healthy lifestyle factors in relation to the risk of $\mathrm{BC}$ among Iranian adult women.

\section{Methods}

\section{Research design and methods}

This project is a population-based case-control study on women aged above 30 years. Patients with $\mathrm{BC}$ were recruited if disease status was diagnosed during the maximum of last six-month by physical examination and mammography findings. Patients were recruited from those referred to hospitals or private clinics who were undergoing surgical resection of $\mathrm{BC}$, chemotherapy or radiotherapy, or all of them. $\mathrm{BC}$ was defined as primary incident breast tumor with invasive behavior which its histology was available from medical registered history. We did not include patients with a history of any type of neoplastic lesion or cysts (exception of current BC) as well as those with a history of any hormone replacement therapy. As hormone use is not part of lifestyle, we did not consider it in our lifestyle score construction. In addition, those who were on a special diet were not included in this study. Age-matched controls were selected from healthy women, who have no relationship with $\mathrm{BC}$ patients or had no family history of BC. In addition to age, we did our best to match controls in terms of socioeconomic status (SES) with the cases. Controls meeting our inclusion criteria (female, Iranian nationality, no history of any malignancy, cysts and medical disorder, having no special diet or hormone replacement therapy) were selected. Controls were randomly selected from apparently healthy women by multistage cluster random sampling method. Individuals who were not relatives of patients with BC that attended primary health care centers for their annual personal checkup or attended to receive required information about their children (i.e. growth monitoring, vaccination, ...) were selected. From several healthcare centers in Isfahan, we randomly chose two centers. First, considering the population under coverage, and then based on attendance of women to these centers, required sample were recruited. Eligible subjects culminated to 350 cases and 700 controls. Written informed consents were obtained from all subjects.

\section{Assessment of diet}

Dietary data was collected using a 106-item Willettformat semi-quantitative dish-based food frequency questionnaire (FFQ) which was designed and validated specifically for Iranian adults. We computed daily intakes of all food items and then converted them to grams per day using household measures [15]. The validity of the questionnaire as well as the detailed information about design and foods included has been reported previously [16]. Briefly, it is based on frequency of consumption of food along the last year in addition to common portion sizes in Iran. The daily value for each item was calculated according to food composition, average of reported frequency, and specified portion size. As for nutrient intakes, it was calculated by adding the nutrient contents of all food and dishes. The nutrient intake for each participant was obtained by Nutritionist IV software, which was modified for Iranian foods. In general, FFQ provides valid and reliable measures of the average intake of foods [17], food groups [17], and nutrients [18] over the long-term.

\section{Assessment of physical activity}

According to an interview-based International Physical activity questionnaire, data on PA was obtained by using participants oral responses, and is expressed as metabolic equivalent h/wk. (MET h/wk) [19]. The questionnaire included queries on five activity domains: job-related PA; transportation PA; activities for housework, and house maintenance; recreation, sports, and leisure-time PA; and time spent sitting. We asked participants to consider all the vigorous and moderate activities as well as the time spent during the last seven days. 


\section{Assessment of smoking}

Smoking was examined using a pretested self-administered questionnaire. The question to the participants was "Are you a smoker or not a smoker or an ex-smoker?". Since the prevalence of smoking in our study population was low, we assumed participants as "smokers" or "nonsmokers". In the current study, participants who have reported smoking were defined as smokers, and those who reported as nonsmokers or ex-smokers were considered as "nonsmokers".

\section{Assessment of healthy lifestyle score}

To construct a healthy lifestyle score, we used data from dietary intakes, PA and smoking status. For the assessment of a healthy dietary intake, we used the previously designed HEI-2010 [20]. The index is constructed of 12 components (total and whole fruits, total vegetables, greens and beans, whole grains, dairy, total protein foods, seafood and plant proteins, fatty acids, refined grains, sodium, and empty calories). Alcohol consumption was not involved in our study due to the absence of information in the original dataset. In the construction of index, first we calculated the energy adjusted intakes of the HEI-2010 components by the residual method [20]. Second, based on the deciles categories of energy adjusted intakes of these components, classification of participants was performed. The usage of decile categories of components instead of quantitative classifications was considered since scoring by deciles would be least disposed to misclassification. Participants in the highest deciles of fruits, vegetables, whole grains, nuts and legumes, long chain omega- 3 fats and polyunsaturated fatty acids were given the score of 10 , whereas those in the lowest deciles of these items were given the score of 1. Participants in the other deciles of these components were given the corresponding scores. Concerning sugar sweetened drinks and fruit juice, red and processed meat, trans fat, sodium intake, added sugars and saturated fatty acids, the lowest deciles were given a score of 10, whereas the highest deciles were given the score of 1 . Individuals in deciles $9,8,7,6,5,4,3$ and 2 of these components were given the scores of $2,3,4,5,6,7,8$ and 9, respectively. Then to calculate the HEI-2010, we summed up the scores for the individual items, resulting in a minimum score of 10 and a maximum score of 100 . Individuals who were in the upper two fifths of HEI2010 were considered to have a healthy diet. As for PA, low risk groups were defined as participants with active and moderately active lifestyle. Regarding cigarette smoking, low risk groups were defined as ex-smokers and individuals who never smoked. As for the HLS development, participants were scored on three modifiable lifestyle factors as unhealthy (zero points) or healthy (one point). Participants obtained one point for each respective lifestyle factor: nonsmoking, adherence to healthy dietary intake, or regular PA. A combined score (zero to three points) was obtained by summing up the scores of the three factors (Fig. 1).

\section{Assessment of other variables}

To measure SES of participants, the socio-economic questionnaire constituting of categorized items on three groups of low, middle and high SES was completed. Also, we evaluated the anthropometric indices of subjects. Measurement of weight was done by digital scales while subjects were minimally clothed and not wearing shoes. Weight was recorded to the nearest $100 \mathrm{~g}$. Height was assessed by using a tape measure while the subjects are not wearing shoes, standing, and shoulders in normal position. By the help of a self-reported questionnaire, we obtained the data on weight (in kilograms) and height (in centimeters). Body mass index (BMI) was calculated by dividing the weight in kilograms by square of height in meters. Participants were classified into two groups based on their BMI: normal weight $\left(18.5-24.9 \mathrm{~kg} / \mathrm{m}^{2}\right)$, overweight or obese $\left(>25.0 \mathrm{~kg} / \mathrm{m}^{2}\right)$. Waist circumference was taken at the narrowest level, and hip circumference was measured at the maximum level over light clothing by the aid of an unstretched tape measure without any pressure to body surface. Measurements were calculated to the nearest $0.1 \mathrm{~cm}$.

\section{Statistical analysis}

Before starting the analysis, we found that number of individuals in the lowest category of HLS (i.e. HLS $=0$ ) was very low $(n=22)$. To avoid having wide confidence intervals (CIs) in this category, we decided to merge the lowest two categories of HLS (0 and 1) and consider them as one category. General characteristics and dietary intakes of cases and controls were assessed using independent samples Student's $t$ test for continuous variables and chi-square for categorical variables. General characteristics and dietary intakes of study participants across categories of the HLS were examined using one-way analysis of variance (ANOVA) for continuous variables and chi-square for categorical variables. The association of HLS with $\mathrm{BC}$ was assessed by using conditional logistic regression in different models. Age (continuous), residence (urban/rural), marital status (non/married/not married), SES (poor/middle/high class), education (educated/not educated), family history of BC (yes/no), menopausal status (yes/no), breast feeding (yes/no), history of disease (yes/no) and supplement use (yes/no) were adjusted in the first model. BMI was additionally adjusted in the second model. To determine the association of individual components of HLS with BC risk, we constructed crude and multivariable-adjusted models controlling for above mentioned covariates, as well as other components of HLS for each component. In 


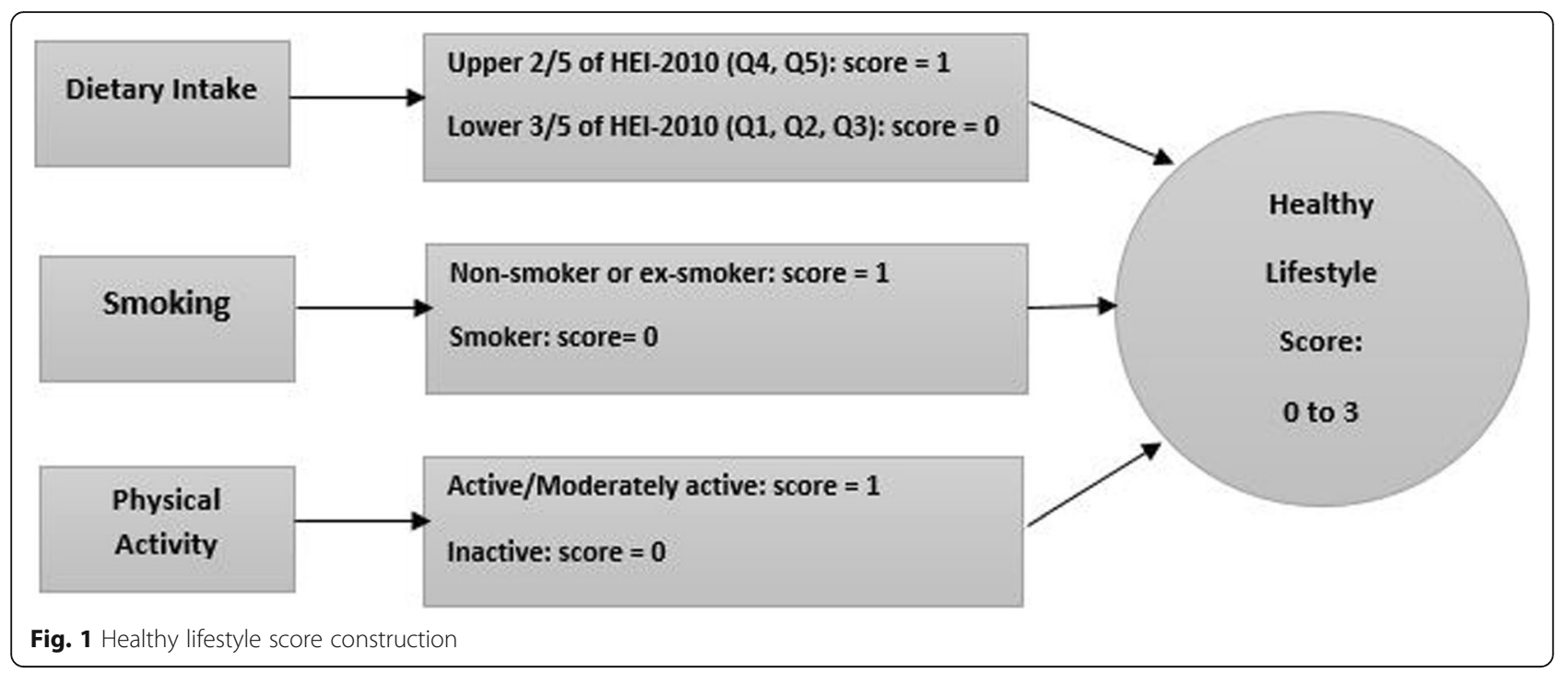

addition to the whole study population, the analyses were also stratified by menopausal status. In these analyses, all above-mentioned covariates were considered except for menopausal status. All confounders were chosen based on previous publications. The statistical analyses were carried out by using IBM SPSS statistics 25. Significance level was considered at $P<0.05$.

\section{Results}

\section{General characteristics of study participants}

General characteristics of study participants with and without BC are presented in Table 1. Participants with $\mathrm{BC}$ were more likely to be older, have a family history of
$\mathrm{BC}$, and menopausal than their controls. In contrary, they were less likely to be married, educated, and had a lower mean BMI than controls. The prevalence of smoking was higher among patients with $\mathrm{BC}$ than controls. No other significant difference was seen in terms of other general characteristics comparing individuals with and without $\mathrm{BC}$.

As for the distribution of participants in terms of general characteristics across categories of HLS, subjects with the highest score were more likely to be residing in urban region, educated, have a higher BMI and physically active, and less likely to have poor SES than those with the lowest score. In the highest HLS, no subjects

Table 1 General characteristics of study participants

\begin{tabular}{|c|c|c|c|c|c|c|c|}
\hline & Groups & & & Healthy Life & Score & & \\
\hline & Cases $n=350$ & Controls $n=700$ & $P^{*}$ & $1 n=185$ & $2 n=455$ & $3 n=410$ & $P^{*}$ \\
\hline Age (year) & $65 \pm 11$ & $61 \pm 10$ & $<0.001$ & $64 \pm 11$ & $62 \pm 10$ & $62 \pm 11$ & 0.065 \\
\hline Residing in Urban Region & $126(36)$ & $252(36)$ & 0.964 & $44(24)$ & $150(33)$ & $184(45)$ & $<0.001$ \\
\hline Married & $262(75)$ & $616(88)$ & $<0.001$ & $153(83)$ & $382(84)$ & $340(83)$ & 0.650 \\
\hline Educated & $59(17)$ & $203(29)$ & $<0.001$ & $27(15)$ & $100(22)$ & $135(33)$ & $<0.001$ \\
\hline Family History of BC & $31(9)$ & $21(3)$ & $<0.001$ & $16(9)$ & $22(5)$ & $16(4)$ & 0.079 \\
\hline Smoker & $59(17)$ & $91(13)$ & 0.055 & $86(47)$ & $63(14)$ & 0 & $<0.001$ \\
\hline Post-menopause & $308(88)$ & $539(77)$ & $<0.001$ & $151(82)$ & $368(81)$ & $328(80)$ & 0.930 \\
\hline BMI $\left(\mathrm{kg} / \mathrm{m}^{2}\right)$ & $22 \pm 5$ & $26 \pm 5$ & $<0.001$ & $3 \pm 5$ & $24 \pm 5$ & $25 \pm 5$ & $<0.001$ \\
\hline Physical Activity (MET-hr/wk) & $35 \pm 7$ & $35 \pm 7$ & 0.196 & $30 \pm 6$ & $34 \pm 7$ & $38 \pm 5$ & $<0.001$ \\
\hline Breast Feeding & $119(34)$ & $238(34)$ & 0.926 & $61(33)$ & $145(32)$ & $151(37)$ & 0.298 \\
\hline Poor Social Economic Status & $115(33)$ & $203(29)$ & 0.311 & $79(43)$ & $150(33)$ & $90(22)$ & $<0.001$ \\
\hline History of Disease & $35(10)$ & $63(9)$ & 0.407 & $18(10)$ & $50(11)$ & $24(6)$ & 0.026 \\
\hline Supplement Use & $31(9)$ & $70(10)$ & 0.715 & $20(11)$ & $45(10)$ & $36(9)$ & 0.575 \\
\hline
\end{tabular}

Variables: Age, BMI and PA are Means \pm SD, other variable values are counts, values between () are \%.

* $P$ values were obtained from independent Student's t test, one-way ANOVA or $X 2$ test, where appropriate 
were smokers versus $47 \%$ in the lowest. No other significant differences were found between categories of HLS in terms of other general characteristics (Table 1).

\section{Dietary intakes of study participants}

Dietary intakes of study participants with and without BC across categories of HLS are provided in Table 2. Compared to controls, individuals with $\mathrm{BC}$ had higher intakes of total energy, carbohydrates, fats, saturated fats, monounsaturated fatty acids (MUFAs), trans fats, cholesterol, red and processed meat, salt, sugar sweetened beverage, and lower intakes of polyunsaturated fatty acids (PUFAs). On micronutrient level, individuals with $\mathrm{BC}$ had higher intakes of vitamin $\mathrm{E}$, vitamin $\mathrm{C}$, potassium, calcium, zinc, iron and magnesium. On HEI-2010 food groups level, individuals with $\mathrm{BC}$ had higher intakes of empty calories, whole fruits and dairy. In contrast, they had less intakes fruit juice, total vegetables, legumes, and lower FA ratio. A marginally significant association was found between $B C$ and higher intakes of sea food, eggs, and vitamin B12 with no difference in vitamin B6 intake between cases and controls or copper intake.

Comparison of participants according to their dietary intakes across categories of HLS is shown in Table 2. On macronutrient level, individuals in the highest category of HLS had higher intake of MUFAs, PUFAs, cholesterol, total fiber, white meat, red and processed meat, but lower intakes of saturated fat, trans FA, and salt compared with those in the lowest category of HLS.

On micronutrient level, individuals in the highest category of HLS had higher intakes of vitamin A, vitamin C, vitamin E, vitamin B6, potassium, calcium, zinc, selenium, vitamin b12, iron, magnesium, and copper.

On HEI 2010 food groups level, individuals in the highest category of HLS had higher intakes of fruit juice, whole fruit, total vegetables, total protein, legumes, dairy, seafood, and higher FA ratio but lower intake of empty calories. A marginally significant association was revealed between having a higher HLS $(\mathrm{HLS}=3)$ and higher intakes of vitamin $\mathrm{D}$ and folate compared to lowest category of HLS (HLS $=1)$.

\section{Association between HLS and odds of BC}

Multivariable-adjusted odd ratios for BC across categories of HLS is shown in Table 3. Participants with the highest HLS had significantly lower odds of BC (OR: 0.45 ; $95 \%$ CI: $0.31,0.65)$ than those with the lowest score. After adjustment for potential confounding variables, participants with the highest HLS were $52 \%$ less likely to have $\mathrm{BC}$ compared with those with the lowest score (OR: 0.48; 95\% CI: 0.32, 0.71). Further adjustment for BMI did not affect our findings; individuals with the highest score were 0.61 times less likely to have $\mathrm{BC}$ than those with the lowest score (OR: 0.62; 95\% CI: 0.40, 0.94, $P_{\text {trend }}=0.01$ ).

The association of HLS and BC was also examined stratified by menopausal status (Table 3). Among postmenopausal women, women with the highest HLS had $58 \%$ lower odds of $\mathrm{BC}$ compared with those with the lowest score (OR: 0.42; 95\% CI: 0.28, 0.63, $P_{\text {trend }}<0.001$ ). This inverse association remained significant after further adjustment for potential confounding variables (OR: 0.45; 95\% CI: $0.23,0.69, P$ trend $<0.001$ ) and even after controlling for BMI (OR: 0.56; 95\% CI: $0.36,0.88, P$ trend $=0.004)$. We found no significant association between HLS score and odds of BC in premenopausal women in crude or adjusted models.

Model I: Adjusted for age, residence, marital status, SES, education, family history of $\mathrm{BC}$, menopausal status, breast feeding, history of disease and supplement use. Model II: Further controlled for BMI.

\section{Association between $\mathrm{HEl}$ and odds of $\mathrm{BC}$}

The association between Healthy Eating Index and breast cancer are shown in Table 4. Participants with the greatest adherence to HEI-2010 recommendations (highest HEI score) had significantly lower odds of BC (OR: 0.40 ; 95\% CI: $0.27,0.57$ ) than those with the lowest score. After adjustment for potential confounding variables, participants with the healthier diet (highest HEI score) were $60 \%$ less likely to have $\mathrm{BC}$ compared with those with less healthy diet (lowest HEI score) (OR: 0.40; 95\% CI: 0.26, 0.56). This association remained significant even after adjustment for BMI (OR: 0.54; 95\% CI: 0.35, 0.82, $P_{\text {trend }}<$ 0.001 ). When we analyzed data stratified by menopausal status (Table 4), we found that postmenopausal women with the greatest adherence to HEI2010 (highest HEI score) had 61\% lower odds of BC compared with those with the lowest HEI score) (OR: 0.39; 95\% CI: 0.26, 0.56, $P_{\text {trend }}<0.001$ ). This result remained significant after further adjustment for potential confounding variables (OR: $0.40 ; 95 \% \mathrm{CI}$ : 0.26, 0.62, $P$ trend $<0.001)$ and even after controlling for BMI (OR: 0.50; 95\% CI: 0.32, 0.79, $P$ trend $=$ 0.004). Neither in crude nor in adjusted models, was any association between HEI score and odds of BC in premenopausal women.

\section{Association between physical activity and odds of $B C$}

Multivariable-adjusted odds ratios for $\mathrm{BC}$ across categories of PA are shown in Table 5. The association was also examined by menopausal status. Neither in crude nor in adjusted models, we found no significant association between PA and odds of $\mathrm{BC}$ in the whole population as well as in post- or pre-menopausal women. 
Table 2 Dietary intakes of study participants

\begin{tabular}{|c|c|c|c|c|c|c|c|}
\hline & \multicolumn{3}{|l|}{ Groups } & \multicolumn{4}{|c|}{ Healthy Lifestyle Score } \\
\hline & \multirow{2}{*}{$\begin{array}{l}\text { Cases }(n=350) \\
\text { Mean } \pm \text { SD }\end{array}$} & \multicolumn{2}{|l|}{ Controls $(n=700)$} & \multirow{2}{*}{$\begin{array}{l}1(n=185) \\
\text { Mean } \pm S D\end{array}$} & \multirow{2}{*}{$\begin{array}{l}2(n=455) \\
\text { Mean } \pm S D\end{array}$} & \multicolumn{2}{|l|}{$3(n=410)$} \\
\hline & & Mean \pm SD & $P^{*}$ & & & Mean \pm SD & $P^{*}$ \\
\hline Total energy (kcal/d) & $2495 \pm 793$ & $2178 \pm 608$ & $<0.001$ & $2196.9 \pm 700.1$ & $2288 \pm 721$ & $2321 \pm 653$ & 0.126 \\
\hline \multicolumn{8}{|l|}{ Micronutrients } \\
\hline Vitamin A (IU/d) & $3277 \pm 2941$ & $3093 \pm 2555$ & 0.297 & $2160 \pm 1731$ & $2968 \pm 2534$ & $3810 \pm 3027$ & $<0.001$ \\
\hline Vitamin D (IU/d) & $53 \pm 242$ & $33 \pm 51$ & 0.145 & $21 \pm 27$ & $50 \pm 216$ & $38 \pm 47$ & 0.078 \\
\hline Vitamin E (IU/d) & $7 \pm 3$ & $6 \pm 3$ & 0.008 & $6 \pm 3$ & $6 \pm 3$ & $7 \pm 4$ & $<0.001$ \\
\hline Vitamin C (mg/d) & $69 \pm 45$ & $58 \pm 38$ & $<0.001$ & $49 \pm 37$ & $59 \pm 38$ & $71 \pm 43$ & $<0.001$ \\
\hline Vitamin $B_{6}(\mathrm{mg} / \mathrm{d})$ & $2 \pm 1$ & $2 \pm 0.5$ & 0.052 & $1 \pm 0.5$ & $1.6 \pm 0.5$ & $1.7 \pm 0.5$ & $<0.001$ \\
\hline Folate (mcg/d) & $601 \pm 206$ & $580 \pm 189$ & 0.103 & $560 \pm 180$ & $585 \pm 202$ & $602 \pm 191$ & 0.053 \\
\hline Vitamin $B_{12}(\mathrm{mcg} / \mathrm{d})$ & $3 \pm 3$ & $2.6 \pm 2$ & 0.056 & $2 \pm 3$ & $3 \pm 2$ & $3 \pm 2$ & 0.001 \\
\hline Potassium (mg/d) & $3071 \pm 1128$ & $2850 \pm 818$ & 0.001 & $2669 \pm 882$ & $2895 \pm 990$ & $3069 \pm 876$ & $<0.001$ \\
\hline Calcium (mg/d) & $821 \pm 331$ & $736 \pm 276$ & $<0.001$ & $643 \pm 276$ & $761 \pm 309$ & $824 \pm 279$ & $<0.001$ \\
\hline Zinc (mg/d) & $10.5 \pm 3$ & $10 \pm 3$ & 0.017 & $9 \pm 3$ & $10 \pm 3$ & $11 \pm 3$ & $<0.001$ \\
\hline Copper (mg/d) & $2 \pm 1$ & $2 \pm 0.5$ & 0.086 & $1.6 \pm 1$ & $1.7 \pm 1$ & $1.8 \pm 0.5$ & 0.002 \\
\hline Selenium (mcg/d) & $148 \pm 52$ & $146 \pm 52$ & 0.493 & $135 \pm 51$ & $145 \pm 54$ & $153 \pm 51$ & $<0.001$ \\
\hline Iron (mg/d) & $18 \pm 6$ & $17 \pm 5$ & 0.009 & $16 \pm 5$ & $17 \pm 6$ & $18 \pm 5$ & 0.001 \\
\hline Magnesium (mg/d) & $472 \pm 156$ & $442 \pm 141$ & 0.002 & $428 \pm 145$ & $449 \pm 153$ & $466 \pm 139$ & 0.010 \\
\hline \multicolumn{8}{|l|}{ Food Groups } \\
\hline Carbohydrates (g/d) & $340 \pm 123$ & $306 \pm 97$ & $<0.001$ & $318 \pm 112$ & $317 \pm 113$ & $317 \pm 99$ & 0.994 \\
\hline Proteins (g/d) & $79 \pm 30$ & $77 \pm 28$ & 0.381 & $66 \pm 25$ & $77 \pm 31$ & $84 \pm 26$ & $<0.001$ \\
\hline Red and processed meat $(\mathrm{g} / \mathrm{d})$ & $13 \pm 201$ & $10 \pm 12$ & 0.002 & $8 \pm 10$ & $11 \pm 15$ & $12 \pm 18$ & 0.006 \\
\hline White meat $(\mathrm{g} / \mathrm{d})$ & $71 \pm 90$ & $74 \pm 68$ & 0.478 & $52 \pm 59$ & $72 \pm 89$ & $84 \pm 63$ & $<0.001$ \\
\hline $\operatorname{Egg}(\mathrm{g} / \mathrm{d})$ & $13 \pm 19$ & $10 \pm 12$ & 0.050 & $10 \pm 17$ & $10 \pm 15$ & $12 \pm 14$ & 0.097 \\
\hline Salt $(g / d)$ & $3.5 \pm 3$ & $2.7 \pm 2$ & $<0.001$ & $3.5 \pm 3$ & $3.1 \pm 3$ & $2.6 \pm 2$ & $<0.001$ \\
\hline Fats $(\mathrm{g} / \mathrm{d})$ & $99 \pm 42$ & $78 \pm 28$ & $<0.001$ & $80 \pm 35$ & $85 \pm 37$ & $86 \pm 32$ & 0.109 \\
\hline Saturated fats (g/d) & $44 \pm 33$ & $26 \pm 20$ & $<0.001$ & $35 \pm 23$ & $34 \pm 30$ & $28 \pm 23$ & $<0.001$ \\
\hline Monounsaturated fats (MUFAs) (g/d) & $21 \pm 9$ & $20 \pm 8$ & 0.011 & $18 \pm 8$ & $20 \pm 8$ & $22 \pm 8$ & $<0.001$ \\
\hline Polyunsaturated fats (PUFAs) (g/d) & $9 \pm 4$ & $10 \pm 8$ & $<0.001$ & $8 \pm 5$ & $9 \pm 5$ & $12 \pm 9$ & $<0.001$ \\
\hline Trans FA (g/d) & $0.3 \pm 0.3$ & $0.2 \pm 0.2$ & $<0.001$ & $0.5 \pm 0.3$ & $0.4 \pm 0.3$ & $0.4 \pm 0.3$ & 0.004 \\
\hline Cholesterol (mg/d) & $205 \pm 134$ & $182 \pm 97$ & 0.005 & $152 \pm 105$ & $184 \pm 113$ & $212 \pm 107$ & $<0.001$ \\
\hline Sugar sweetened beverages (g/d) & $40 \pm 83$ & $22 \pm 28$ & $<0.001$ & $33 \pm 63$ & $28 \pm 52$ & $25 \pm 52$ & 0.265 \\
\hline Total fiber (g/d) & $23 \pm 8$ & $22 \pm 8$ & 0.328 & $20 \pm 7$ & $22 \pm 8$ & $24 \pm 8$ & $<0.001$ \\
\hline \multicolumn{8}{|l|}{ HEl-2010 Food Groups } \\
\hline Fruit Juice (g/d) & $1 \pm 3$ & $2 \pm 4$ & 0.002 & $1 \pm 1$ & $1 \pm 3$ & $2 \pm 4$ & $<0.001$ \\
\hline Whole Fruit (g/d) & $215 \pm 195$ & $140 \pm 122$ & $<0.001$ & $133 \pm 148$ & $154 \pm 146$ & $191 \pm 162$ & $<0.001$ \\
\hline Total Vegetables (g/d) & $69 \pm 67$ & $87 \pm 75$ & $<0.001$ & $53 \pm 43$ & $75 \pm 76$ & $99 \pm 75$ & $<0.001$ \\
\hline Sea food $(g / d)$ & $12 \pm 51$ & $6 \pm 12$ & 0.066 & $3 \pm 7$ & $10 \pm 44$ & $8 \pm 17$ & 0.023 \\
\hline Total Protein (g/d) & $85 \pm 74$ & $89 \pm 69$ & 0.430 & $69 \pm 62$ & $84 \pm 77$ & $101 \pm 65$ & $<0.001$ \\
\hline Legumes (g/d) & $13 \pm 13$ & $15 \pm 16$ & 0.032 & $11 \pm 9$ & $14 \pm 12$ & $17 \pm 19$ & $<0.001$ \\
\hline Dairy (g/d) & $257 \pm 174$ & $219 \pm 143$ & $<0.001$ & $177 \pm 140$ & $234 \pm 166$ & $253 \pm 143$ & $<0.001$ \\
\hline Whole grains (g/d) & $325 \pm 150$ & $313 \pm 157$ & 0.197 & $311 \pm 150$ & $313 \pm 157$ & $323 \pm 154$ & 0.563 \\
\hline Refined grains (g/d) & $115 \pm 86$ & $115 \pm 74$ & 0.887 & $115 \pm 71$ & $120 \pm 83$ & $109 \pm 76$ & 0.151 \\
\hline
\end{tabular}


Table 2 Dietary intakes of study participants (Continued)

\begin{tabular}{|c|c|c|c|c|c|c|c|}
\hline & \multicolumn{3}{|l|}{ Groups } & \multicolumn{4}{|c|}{ Healthy Lifestyle Score } \\
\hline & Cases $(n=350)$ & Controls $(n=700)$ & & $1(n=185)$ & $2(n=455)$ & $3(n=410)$ & \\
\hline & Mean \pm SD & Mean \pm SD & $P^{*}$ & Mean \pm SD & Mean \pm SD & Mean \pm SD & $P^{*}$ \\
\hline Sodium (mg/d) & $4979 \pm 1867$ & $4795 \pm 1711$ & 0.123 & $4839 \pm 1758$ & $4834 \pm 1753$ & $4890 \pm 1787$ & 0.889 \\
\hline FA ratio & $1 \pm 0.4$ & $1 \pm 0.5$ & $<0.001$ & $1 \pm 0.4$ & $1 \pm 0.4$ & $1 \pm 1$ & $<0.001$ \\
\hline Empty calories (kcal/d) & $149 \pm 132$ & $83 \pm 56$ & $<0.001$ & $127 \pm 124$ & $106 \pm 88$ & $95 \pm 84$ & 0.001 \\
\hline
\end{tabular}

*Obtained by ANOVA

*Ratio of poly- and monounsaturated fatty acids (PUFAs and MUFAs) to saturated fatty acids (SFAs)

\section{Association between smoking and odds of $B C$}

Multivariable-adjusted odds ratios for $\mathrm{BC}$ across categories of smoking are shown in Table 6. Smokers were $41 \%$ more likely to have $\mathrm{BC}$ than non-smokers (OR: 1.41; 95\% CI: 0.99, 2.01, $P$ trend $=0.055)$. However, after adjustment for potential confounding variables, this association disappeared. Neither in crude nor in adjusted models, we found no significant association between smoking and BC in pre- or postmenopausal women.

\section{Discussion}

In this case-control study, we found a significant inverse association between adherence to HLS as well as HEI2010 and odds of BC. This association remained significant after adjusting for several confounding variables. In our stratified analysis by menopausal status, the association remained significant among postmenopausal women, while no association was found among premenopausal group. In terms of components of HLS, no association was found between smoking and PA and odds of $\mathrm{BC}$. To our knowledge, this is the first study to investigate the association between HLS and the risk of $\mathrm{BC}$ in a Middle Eastern country.

$\mathrm{BC}$ is the most prevalent cancer among women worldwide. Healthy lifestyle modification is an important factor in $\mathrm{BC}$ prevention. Earlier studies have shown that at least 25 to $30 \%$ of $\mathrm{BC}$ cases could be prevented if healthy lifestyle was chosen [21]. In the current study, we found that adhering to HLS was associated with a reduced odd of BC. Our findings were in line with previous reports that found an inverse association between HLS and BC. In the Women's Health Initiative Study, postmenopausal women in the highest quintile of Healthy Lifestyle Index (HLI) had a 30\% decreased risk of $\mathrm{BC}$ compared to women in the lowest quintile (HR: 0.70; 95\% CI: 0.64-0.76). In addition, a 4\% decreased risk of $\mathrm{BC}$ was found per unit increase in HLI score (HR: 0.96; 95\% CI: 0.95-0.97) [21]. In the EPIC study, there was a $3 \%$ reduction in the risk of $\mathrm{BC}$ per point increase of the Healthy Lifestyle Index Score (HLIS) among postmenopausal women [22]. In addition, Gemert et al. showed that modifiable risk factors were directly responsible for about one out of four postmenopausal BC cases. Modifiable risk factors composed of BMI, physical inactivity, alcohol consumption, smoking and low dietary fiber intake [23]. In the E3N cohort, adherence of postmenopausal women to a healthy lifestyle by following the recommendations for smoking, BMI, alcohol consumption, fruit and vegetable consumption, and PA, prevented $\mathrm{BC}$ by $6.3 \%$ [24]. However, an increased risk of

Table 3 Multivariable-adjusted ratios for BC across different categories of the Healthy lifestyle (HLS) score

\begin{tabular}{|c|c|c|c|c|c|c|}
\hline \multirow[t]{2}{*}{ HLS range } & \multirow{2}{*}{$\begin{array}{l}1(n=185) \\
\text { OR }\end{array}$} & \multicolumn{2}{|c|}{$2(n=455)$} & \multicolumn{2}{|c|}{$3(n=410)$} & \multirow[t]{2}{*}{$P$ trend } \\
\hline & & OR & $95 \% \mathrm{Cl}$ & OR & $95 \% \mathrm{Cl}$ & \\
\hline \multicolumn{7}{|c|}{ All population } \\
\hline Crude & 1.00 & 0.78 & $0.55-1.11$ & 0.45 & $0.31-0.65$ & $<0.001$ \\
\hline Model I & 1.00 & 0.85 & $0.59-1.22$ & 0.48 & $0.32-0.71$ & $<0.001$ \\
\hline Model II & 1.00 & 0.93 & $0.63-1.38$ & 0.62 & $0.4-0.94$ & 0.011 \\
\hline \multicolumn{7}{|c|}{ Pre-menopause } \\
\hline Crude & 1.00 & 1 & $0.33-2.55$ & 0.63 & $0.23-1.7$ & 0.278 \\
\hline Model I & 1.00 & 0.98 & $0.36-2.66$ & 0.67 & $0.23-1.99$ & 0.386 \\
\hline Model II & 1.00 & 1.05 & $0.34-3.19$ & 1.59 & $0.45-5.59$ & 0.478 \\
\hline \multicolumn{7}{|c|}{ Post-menopause } \\
\hline Crude & 1.00 & 0.75 & $0.51-1.1$ & 0.42 & $0.28-0.63$ & $<0.001$ \\
\hline Model I & 1.00 & 0.83 & $0.55-1.23$ & 0.45 & $0.23-0.69$ & $<0.001$ \\
\hline Model II & 1.00 & 0.91 & $0.6-1.39$ & 0.56 & $0.36-0.88$ & 0.004 \\
\hline
\end{tabular}


Table 4 Multivariable-adjusted ratios for BC across different categories of the Healthy Eating Index-2010 Score (HEl-2010)

\begin{tabular}{|c|c|c|c|c|c|c|c|c|}
\hline \multirow[t]{2}{*}{ HEI score range } & \multirow{2}{*}{$\begin{array}{l}1(n=254) \\
\text { OR }\end{array}$} & \multicolumn{2}{|c|}{$2(n=277)$} & \multicolumn{2}{|c|}{$3(n=248)$} & \multicolumn{2}{|c|}{$4(n=271)$} & \multirow[t]{2}{*}{$\mathrm{P}$ trenc } \\
\hline & & $\mathrm{OR}$ & $95 \% \mathrm{Cl}$ & $\mathrm{OR}$ & $95 \% \mathrm{Cl}$ & OR & $95 \% \mathrm{Cl}$ & \\
\hline \multicolumn{9}{|l|}{ All population } \\
\hline Crude & 1.00 & 0.60 & $0.42-0.85$ & 0.25 & $0.17-0.37$ & 0.40 & $0.27-0.57$ & $<0.001$ \\
\hline Model I & 1.00 & 0.56 & $0.39-0.81$ & 0.25 & $0.16-0.38$ & 0.40 & $0.26-0.56$ & $<0.001$ \\
\hline Model II & 1.00 & 0.60 & $0.41-0.89$ & 0.33 & $0.21-0.51$ & 0.54 & $0.35-0.82$ & $<0.001$ \\
\hline \multicolumn{9}{|l|}{ Pre-menopause } \\
\hline Crude & 1.00 & 0.16 & $0.05-0.51$ & 0.63 & $0.26-1.5$ & 0.12 & $0.12-0.91$ & 0.129 \\
\hline Model I & 1.00 & 0.15 & $0.04-0.52$ & 0.70 & $0.26-1.86$ & 0.37 & $0.11-1.22$ & 0.238 \\
\hline Model II & 1.00 & 0.12 & $0.03-0.52$ & 1.25 & $0.38-4.15$ & 1.85 & $0.40-8.5$ & 0.44 \\
\hline \multicolumn{9}{|l|}{ Post-menopause } \\
\hline Crude & 1.00 & 0.69 & $0.47-1.01$ & 0.19 & $0.12-0.31$ & 0.39 & $0.26-0.56$ & $<0.001$ \\
\hline Model I & 1.00 & 0.66 & $0.44-0.10$ & 0.19 & $0.12-0.31$ & 0.40 & $0.26-0.62$ & $<0.001$ \\
\hline Model II & 1.00 & 0.68 & $0.45-1.03$ & 0.25 & $0.15-0.40$ & 0.50 & $0.32-0.79$ & $<0.001$ \\
\hline
\end{tabular}

Model I: adjusted for age, residence, marital status, SES, education, family history of B. C, menopausal status, breast feeding, history of disease, supplement use, smoking and physical activity. Model II: further controlled for BMI.

breast cancer-specific mortality was found for individuals with healthy behavior index compared to those with a lower healthy lifestyle score, however, the results was not statistically significant [14]. Therefore, it seems that adherence to healthy lifestyle could be used as a good preventive measure to reduce $\mathrm{BC}$.

When we analyzed the data by menopausal status, we found a significant inverse association in postmenopausal women, but not in premenopausal women. The same associations were found in some studies [21-24]. However, some studies have found a significant inverse association among premenopausal women as well [13, 25]. In a case-control study from Morocco, Khalis et al. found a significant association in premenopausal women. However, the same associations were also reached in postmenopausal women [25]. Furthermore, a study from Mexico revealed that HLI was associated with a decreased odds of BC among both pre- and postmenopausal women [13].

One might question why we only included diet, PA, and smoking as lifestyle factors and did not include other factors in the HLS construction. Earlier studies on lifestyle score have mostly used the variables we used in this study. However, some studies have also included BMI in the score, but as being overweight or obese is a result of an unhealthy lifestyle, we preferred not to include it in the score. We did not consider hormone use because it is not part of lifestyle, rather it's a medication use to control hormone levels. In addition, alcohol

Table 5 Multivariable-adjusted ratios for BC across different categories of Physical activity

\begin{tabular}{|c|c|c|c|c|c|c|c|c|}
\hline \multirow[t]{2}{*}{ PA score range } & \multirow{2}{*}{$\begin{array}{l}1(n=260) \\
\text { OR }\end{array}$} & \multicolumn{2}{|c|}{$2(n=264)$} & \multicolumn{2}{|c|}{$3(n=263)$} & \multicolumn{2}{|c|}{$4(n=263)$} & \multirow[t]{2}{*}{$P$ trenc } \\
\hline & & $\mathrm{OR}$ & $95 \% \mathrm{Cl}$ & $\mathrm{OR}$ & $95 \% \mathrm{Cl}$ & $\mathrm{OR}$ & $95 \% \mathrm{Cl}$ & \\
\hline \multicolumn{9}{|l|}{ All population } \\
\hline Crude & 1.00 & 1.27 & $0.88-1.83$ & 1.14 & $0.79-1.65$ & 1.28 & $0.89-1.84$ & 0.286 \\
\hline Model I & 1.00 & 1.34 & $0.91-1.99$ & 1.26 & $0.85-1.87$ & 1.37 & $0.93-2.03$ & 0.165 \\
\hline Model II & 1.00 & 1.31 & $0.87-1.97$ & 1.18 & $0.78-1.79$ & 1.43 & $0.95-2.15$ & 0.165 \\
\hline \multicolumn{9}{|l|}{ Pre-menopause } \\
\hline Crude & 1.00 & 2.65 & $0.97-7.22$ & 1.66 & $0.57-4.86$ & 2.26 & $0.78-6.43$ & 0.255 \\
\hline Model I & 1.00 & 3.18 & $1.08-9.35$ & 1.92 & $0.61-6.03$ & 2.70 & $0.87-8.31$ & 0.183 \\
\hline Model II & 1.00 & 5.86 & $1.61-21.40$ & 3.34 & $0.86-13.05$ & 4.24 & $1.15-15.65$ & 0.092 \\
\hline \multicolumn{9}{|l|}{ Post-menopause } \\
\hline Crude & 1.00 & 1.12 & $0.75-1.66$ & 1.06 & $0.71-1.58$ & 1.13 & $0.77-1.68$ & 0.602 \\
\hline Model I & 1.00 & 1.17 & $0.76-1.80$ & 1.17 & $0.76-1.80$ & 1.22 & $0.80-1.86$ & 0.403 \\
\hline Model II & 1.00 & 1.13 & $0.72-1.77$ & 1.09 & $0.69-1.70$ & 1.26 & $0.81-1.96$ & 0.406 \\
\hline
\end{tabular}

Model I: Adjusted for age, residence, marital status, SES, education, family history of B. C, menopausal status, breast feeding, history of disease, supplement use, smoking and HEl score. Model II: Further controlled for BMI. 
Table 6 Multivariable-adjusted ratios for BC across different categories of Smoking

\begin{tabular}{llllll}
\hline Smoking Categories & \multicolumn{2}{l}{ Non-smoker } & \multicolumn{2}{l}{ Smoker } & P trend \\
& OR & OR & $95 \% \mathrm{Cl}$ & \\
\hline All population & & & & \\
$\quad$ Crude & 1.00 & 1.41 & $0.99-2.01$ & 0.055 \\
Model I & 1.00 & 1.23 & $0.84-1.82$ & 0.286 \\
Model II & 1.00 & 1.01 & $0.67-1.52$ & 0.943 \\
Pre-menopause & & & & \\
$\quad$ Crude & 1.00 & 0.57 & $0.12-2.64$ & 0.474 \\
Model I & 1.00 & 0.47 & $0.09-2.53$ & 0.411 \\
$\quad$ Model II & 1.00 & 0.47 & $0.08-2.71$ & 0.384 \\
Post-menopause & & & & \\
$\quad$ Crude & 1.00 & 1.40 & $0.97-2.03$ & 0.073 \\
Model I & 1.00 & 1.31 & $0.87-1.98$ & 0.193 \\
Model II & 1.00 & 1.08 & $0.70-1.65$ & 0.726 \\
\hline
\end{tabular}

Model I: Adjusted for age, residence, marital status, SES, education, family history of B. C, menopausal status, breast feeding, history of disease, supplement use, HEI score and physical activity. Model II: Further controlled for BMI.

consumption can be considered in the diet part of the scoring but given the culture of the study population and their religion, alcohol consumption is forbidden in the country and we did not collect information on this variable. With regards to breastfeeding and its history among study population, we believe that despite its contribution to the risk of $\mathrm{BC}$, it is not a lifestyle factor. In general, lifestyle constitutes a combination of diet, physical activity, smoking and stress. However, we did not have information on stress in the current study population.

Lack of finding a significant association between HLS and odds of $\mathrm{BC}$ in premenopausal women might be explained by the low number of premenopausal women in the current study (850 post- vs 200 premenopausal women). Another explanation is the variation in components of HLS. In the study by Khalis et al., they used an index composed of diet, PA, BMI, smoking, alcohol consumption, and breastfeeding [25]. In the study by Sánchez-Zamorano et al., their index identified healthy lifestyle as being in the lowest tertile of the Western dietary pattern, never consuming alcohol, smoking less than 100 cigarettes, and practicing moderate and vigorous intensity exercise [13]. We used HEI as a component of healthy diet, while most other studies have used fruit and vegetables as the healthy diet.

There are several mechanisms that explain the inverse association between healthy lifestyle and odds of $\mathrm{BC}$. Healthy diet plays an important role in cancer prevention as shown in the literature. Hormone receptorpositive breast tumor depends on hormonal risk factors for ongoing proliferation; while hormone receptor- negative $\mathrm{BC}$ depends more on non-hormonal risk factors like diet [26]. A healthy diet that is abundant in dietary fiber, antioxidants and vitamins might decrease the proliferation of ER negative BC through suppressing the inflammatory response, neutralizing free radicals and preventing DNA damage. Another plausible mechanism is through weight management, since adiposity have been defined as a risk factor for BC [27]. PA and nonsmoking may also contribute to the protective associations of HLS with chronic conditions; however, their role in the reduced odds of $\mathrm{BC}$ in our study was not prominent.

A healthy lifestyle composing of a healthy diet along with regular PA and nonsmoking behavior has been shown to decrease the risk of BC. Despite lack of finding significant associations between diet and $\mathrm{BC}$ in most previous studies, we found a significant inverse association between adhering to a healthy diet and odds of $\mathrm{BC}$. The main difference between our study and previous studies is using HEI-2010 as an index of healthy diet, while other studies have mostly used some components of the healthy diet instead. Another possible explanation for the difference between our findings and those in previous studies might be attributed to the lack of alcohol consumption in Iranian's society. One additional point that should be considered is high consumption of low fat, rather than high-fat dairy products in Iran compared with other countries. We did not find any significant association between smoking and BC in the current study, in contrast to the results of previous studies. In fact, prevalence of smoking in Iranian women is not so high compared to other countries. Based on Tehran Lipid and Glucose Study (TLGS), the prevalence of smoking in Iranian women is $2.1 \%$ [28]. In addition, we did not find any significant association between PA and BC, though previous studies have shown a significant inverse association between PA and BC. One possible explanation is that most Iranian women are housewives, which might lead to have low PA levels. Meanwhile, the Iranian national study have shown that the prevalence of inactivity in urban and rural areas of women aged 15 years and older was $58.8 \%$ [29]. Given the high prevalence of inactivity, the distribution of this variable might not be normal to let us find a real association. In addition, the IPAQ used in the assessment of PA in the current study might have some limitations, though it was validated in Iran [30].

Our study has several strengths. We used the HEI2010 to assess dietary quality, rather than using some components of the diet. In addition, we accounted for several confounding factors in our analysis. The use of validated FFQ and being the first study examining the link between HLS and odds of BC in a Middle Eastern population would be among others. However, our study 
has some limitations. The case-control design of the study, which is subject to selection and recall bias, prohibited us to confer causality. Measurement errors might result in misclassification of study participants in terms of dietary intakes. The number of postmenopausal women is greater than the number of premenopausal women. Moreover, HEI-2010 is a USA-based index for assessing diet quality which might not be totally applicable to a population with a different diet and lifestyle. Hormone receptor status is very important in studying factors related to $\mathrm{BC}$ as evidenced by previous studies, yet we did not have information about it in the current study which might be an important limitation.

While previous studies in Iran have assessed the association between individual lifestyle factors and $\mathrm{BC}$ risk, no study was done before examining the association between a combined healthy lifestyle score and odds of BC. In addition, limited studies have used HEI-2010 as a dietary assessment tool in their lifestyle score. Our study contributed to the accumulating evidence for adherence to a high HLS or HEI-2010 as being associated with decreased $\mathrm{BC}$ odds in women in general.

\section{Conclusion}

In conclusion, we found a protective association between HLS and odds of breast cancer. This association was particularly seen in postmenopausal women. Therefore, adoption to a healthy lifestyle, including healthy diet, PA and nonsmoking behavior might help prevent the prevalence of breast cancer in the community setting. No significant association was found between PA or nonsmoking behavior with odds of BC. Further studies, however, are needed to confirm our current findings.

\section{Abbreviations}

ANOVA: One-way analysis of variance; BC: Breast Cancer; BMI: body mass index; Cl: Confidence Interval; FFQ: food frequency questionnaire; HEl2010: Healthy Eating Index-2010; HLS: Healthy Lifestyle Score: MUFAs: monounsaturated fatty acids; OR: Odd Ratio; PA: Physical Activity; PUFAs: polyunsaturated fatty acids; SD: Standard Deviation; SES: Social economic status

\section{Acknowledgements}

Not applicable.

\section{Authors' contributions}

BG and AE contributed in conception, design, statistical analyses, data interpretation and manuscript drafting. SBK, SEK, and LA contributed in data collection, interpretation and manuscript drafting. AE supervised the study. All authors approved the final manuscript for submission.

\section{Funding}

Not applicable.

\section{Availability of data and materials}

The datasets used and/or analyzed during the current study are available from the corresponding author on reasonable request.

\section{Ethics approval and consent to participate}

The research project was approved by Tehran University of MedicalSciences, Tehran, Iran Ethics Committee. The purpose of the study was fully explained in the questionnaire and responses to questions was on a voluntary basis. All participants were assured of anonymity and confidentiality.

\section{Consent for publication}

Participants were provided a study overview and verbal consent was attained.

\section{Competing interests}

The authors declare that they have no competing interests.

\section{Author details}

${ }^{1}$ Department of Community Nutrition, School of Nutritional Sciences and Dietetics, Tehran University of Medical Sciences, P.O. Box 14155-6117, Tehran, Iran. ${ }^{2}$ Obesity and Eating Habits Research Center, Endocrinology and Metabolism Molecular- Cellular Sciences Institute, Tehran University of Medical Sciences, Tehran, Iran. ${ }^{3}$ Department of Community Nutrition, School of Nutrition and Food Science, Isfahan University of Medical Sciences, Isfahan, Iran.

Received: 21 August 2019 Accepted: 7 January 2020

Published online: 14 January 2020

\section{References}

1. How diet, nutrition and physical activity affect breast cancer risk. https:// www.wcrf.org/dietandcancer/breast-cancer. Accessed 10 Nov 2019.

2. Farhood B, Geraily G. Alizadeh Alljoph. incidence and mortality of various cancers in Iran and compare to other countries: a review article. Iran J Public Health. 2018;47:309.

3. Forouzanfar $\mathrm{MH}$, Foreman $\mathrm{KJ}$, Delossantos $\mathrm{AM}$, et al. Breast and cervical cancer in 187 countries between 1980 and 2010: a systematic analysis. The Lancet. 2011;378:1461-84.

4. Lauby-Secretan B, Scoccianti C, Loomis D, et al. Body fatness and cancer-viewpoint of the IARC working group. N Engl J Med. 2016;375:794-8.

5. Key J, Hodgson S, Omar RZ, et al. Meta-analysis of studies of alcohol and breast cancer with consideration of the methodological issues. Cancer Causes Control. 2006:17:759-70.

6. Godinho-Mota JCM, Gonçalves LV, Mota JF, et al. Sedentary behavior and alcohol consumption increase breast Cancer risk regardless of menopausal status: a case-control study. Nutrients. 2019;11:1871.

7. Eliassen AH, Missmer SA, Tworoger SS, et al. Endogenous steroid hormone concentrations and risk of breast cancer among premenopausal women. J Natl Cancer Inst. 2006;98:1406-15.

8. Kelsey JL, Gammon MD, John EM. Reproductive factors and breast cancer. Epidemiol Rev. 1993;15:36-47.

9. Kaminska M, Ciszewski T, Lopacka-Szatan K, Miotla P, Staroslawska E. Breast cancer risk factors. Prz Menopauzalny. 2015;14:196-202.

10. Michels KB, Mohllajee AP, Roset-Bahmanyar E, Beehler GP, Moysich KBJCIIJotACS. Diet and breast cancer: a review of the prospective observational studies. Cancer. 2007:109:2712-49.

11. Blanchard CM, Courneya KS, Stein K. Cancer survivors' adherence to lifestyle behavior recommendations and associations with health-related quality of life: results from the American Cancer Society's SCS-II. J Clin Oncol. 2008;26: 2198-204.

12. Arthur R, Kirsh VA, Kreiger N, Rohan T. A healthy lifestyle index and its association with risk of breast, endometrial, and ovarian cancer among Canadian women. Cancer Causes Control. 2018;29:485-93.

13. Sánchez-Zamorano LM, Flores-Luna L, Ángeles-Llerenas A, et al. Healthy lifestyle on the risk of breast cancer. Cancer Epidemiol Biomark Prev. 2011; 20:912-22.

14. Heitz AE, Baumgartner RN, Baumgartner KB, Boone SD. Healthy lifestyle impact on breast cancer-specific and all-cause mortality. Breast Cancer Res Treat. 2018;167:171-81.

15. Ghaffarpour M, Houshiar-Rad A, Kianfar H, Ghaffarpour M. The manual for household measures, cooking yields factors and edible portion of foods. Tehran: Keshavarzi Press. 1999;7:213.

16. Keshteli AH, Esmaillzadeh A, Rajaie S, et al. A dish-based semi-quantitative food frequency questionnaire for assessment of dietary intakes in epidemiologic studies in Iran: design and development. Int J Prev Med. 2014:5:29.

17. Barak F, Falahi E, Keshteli AH, et al. Red meat intake, insulin resistance, and markers of endothelial function among Iranian women. Mol Nutr Food Res. 2015;59:315-22. 
18. Salehi-Abargouei A, Esmaillzadeh A, Azadbakht L, et al. Nutrient patterns and their relation to general and abdominal obesity in Iranian adults: findings from the SEPAHAN study. Eur J Nutr. 2016;55:505-18.

19. Ainsworth BE, Haskell WL, Whitt MC, et al. Compendium of physical activities: an update of activity codes and MET intensities. Med Sci Sports Exerc. 2000;32:5498-504.

20. Willett W, Stampfer MJ. Total energy intake: implications for epidemiologic analyses. Am J Epidemiol. 1986;124(1):17-27.

21. Arthur R, Wassertheil-Smoller S, Manson JE, et al. The combined Association of Modifiable Risk Factors with breast Cancer risk in the Women's Health Initiative. Cancer Prev Res (Phila). 2018;11:317-26.

22. McKenzie $F$, Ferrari $P$, Freisling $H$, et al. Healthy lifestyle and risk of breast cancer among postmenopausal women in the E uropean $\mathrm{P}$ rospective I nvestigation into C ancer and N utrition cohort study. Int J Cancer. 2015; 136:2640-8.

23. van Gemert WA, Lanting $\mathrm{Cl}$, Goldbohm RA, et al. The proportion of postmenopausal breast cancer cases in the Netherlands attributable to lifestyle-related risk factors. Breast Cancer Res Treat. 2015;152:155-62.

24. Dartois L, Fagherazzi G, Boutron-Ruault M-C, Mesrine S, Clavel-Chapelon F. Association between five lifestyle habits and cancer risk: results from the E3N cohort. Eur J Cancer Prev. 2014;7:516-25.

25. Khalis $M$, Chajes V, Moskal A, et al. Healthy lifestyle and breast cancer risk: a case-control study in Morocco. Cancer Epidemiol. 2019;58:160-6.

26. Farsinejad-Marj M, Talebi S, Ghiyasvand R, Miraghajani M. Adherence to Mediterranean diet and risk of breast cancer in premenopausal and postmenopausal women. Arch Iran Med. 2015;18:786-92.

27. Du M, Liu SH, Mitchell C, Fung TT. Associations between diet quality scores and risk of postmenopausal estrogen receptor-negative breast cancer: a systematic review. J Nutr. 2018;148:100-8.

28. Sarraf-Zadegan N, Boshtam M, Shahrokhi S, et al. Tobacco use among Iranian men, women and adolescents. Eur J Pub Health. 2004;14:76-8.

29. Hazavehei SMM, Asadi Z, Hassanzadeh A, Shekarchizadeh P. Comparing the effect of two methods of presenting physical education $\Pi$ course on the attitudes and practices of female students towards regular physical activity in Isfahan University of Medical Sciences. Iran Jo Med Educ. 2008:8:121-31.

30. Moghaddam MB, Aghdam FB, Jafarabadi MA, et al. The Iranian version of international physical activity questionnaire (IPAQ) in Iran: content and construct validity, factor structure, internal consistency and stability. World Appl Sci J. 2012;18:1073-80.

\section{Publisher's Note}

Springer Nature remains neutral with regard to jurisdictional claims in published maps and institutional affiliations.

Ready to submit your research? Choose BMC and benefit from:

- fast, convenient online submission

- thorough peer review by experienced researchers in your field

- rapid publication on acceptance

- support for research data, including large and complex data types

- gold Open Access which fosters wider collaboration and increased citations

- maximum visibility for your research: over $100 \mathrm{M}$ website views per year

At $\mathrm{BMC}$, research is always in progress.

Learn more biomedcentral.com/submissions 